\title{
Asymptotic and Finite User PER Analysis of Successive Interference Cancellation for DS-CDMA
}

\author{
Josep Sala, Senior Member, IEEE, Javier Villares, Member, IEEE, and Francesc Rey, Member, IEEE
}

\begin{abstract}
An expression is derived for the average Packet Error Rate (PER) of a Successive Interference Canceller (SIC) for DS-CDMA when the number of users asymptotically tends to infinity. The asymptotic probability density function of the interference power is governed by a Fokker-Planck differential equation with drift and (asymptotically vanishing) diffusion depending on the PER function of the adopted forward errorcorrecting code (FEC). In addition to the asymptotic solution for the PER, a particle-based algorithm is also developed for computing efficiently the PER in the finite user case.
\end{abstract}

Index Terms-Successive Interference Cancellation, FokkerPlanck equation, Packet Error Rate, CDMA, particle.

\section{IntRoduction AND INITIAL Definitions}

Large $K$-user system analyses [1], [2], [3] have been performed for DS-CDMA and random spreading (long codes ${ }^{1}$ ) in terms of user (Shannon) capacity. We address instead the computation of the asymptotic PER of the SIC multiuser receiver when a specific encoder is shared by all users and exact knowledge of the PER vs. $E_{\mathrm{s}} / N_{\mathrm{t}}$ of the adopted FEC is available, where $E_{\mathrm{s}} / N_{\mathrm{t}}$ stands for the per-user symbol energy to total noise plus interference power spectral density ratio.

Let $K$ and $N$ be the number of active users and spreading factor, respectively. Let $k$ be the user index (with $k=1$ the first cancellation), $E_{s}[k]$ its symbol energy obtained from a given and arbitrary energy profile and $N_{0}$ the one-sided noise power spectral density. The per-user $E_{\mathrm{s}} / N_{\mathrm{t}}$, is given by,

$$
\Gamma_{K}\left[k \mid N_{\mathrm{prv}}\right]=\frac{E_{s}[k]}{N_{0}+N_{\mathrm{prv}}[k]+N_{\mathrm{rem}}[k]}
$$

with $N_{\text {prv }}[k], N_{\text {rem }}[k]$ the residual interference from previous and remaining users at stage $k$, respectively. Assuming that when a user is decoded correctly a residual interference factor $\epsilon$ remains due to imperfect cancellation [4], and denoting $\theta \leq 1$ a generic decorrelation parameter [5] (associated with random inter-user chip- and/or carrier-phase offsets), interferences $N_{\text {prv }}[k], N_{\text {rem }}[k]$ can be written as,

$$
N_{\mathrm{prv}}[k]=\frac{\theta}{N} \sum_{i=1}^{k-1} \epsilon[i] E_{s}[i] ; \quad N_{\text {rem }}[k]=\frac{\theta}{N} \sum_{i=k+1}^{K} E_{s}[i]
$$

where $\epsilon[i] \in\{\epsilon, 1\}$ indicates the successful or unsuccessful decoding at the $i$-th stage, and $\theta / N$ is the average decorrelation factor between signatures of different users in the

This work has been supported by projects 2009SGR1236 (AGAUR), DYNACS (TEC2010- 21245-C02/TCM), COMONSENS (CONSOLIDERINGENIO CSD2008-00010) and THOFU (CENIT CEN-20101019).

The authors are with the Department of Signal Theory and Communications at the Technical University of Catalonia, Barcelona (Spain). E-mail: \{josep.sala,javier.villares,francesc.rey\}@ upc.edu.

${ }^{1}$ pseudo-random codes with period much longer than the symbol period. long code model. Note that, while $N_{\text {rem }}[k]$ is determined by $E_{s}[k], N_{\mathrm{prv}}[k]$ is random due to the sequence of previous packet decoding errors. Then, the SIC operation is modeled as a Markov process of mixed states $s=\{\varepsilon, \xi\}$, with $\varepsilon \in\{0,1\}$ a binary error/non-error state and, $\xi$ the realvalued state modeling the previous residual interference power $N_{\text {prv }}[k]$. Under the long code model, $N_{\text {prv }}[k]$ and $N_{\text {rem }}[k]$ can be assumed Gaussian distributed. Then, the packet error probability at stage $k$, for a given value of $\xi$, is determined by the PER vs. $E_{\mathrm{s}} / N_{\mathrm{t}}$ curve of the adopted FEC,

$$
p_{1}[k \mid \xi] \doteq \operatorname{PER}\left[\Gamma_{K}[k \mid \xi]\right]
$$

Following [3], when $K \rightarrow \infty$, computations in the discrete user index $k \leq K$ are cast into asymptotically equivalent expressions in the continuous variable $t=\lim _{K \rightarrow \infty} \frac{k}{K}(0<$ $t \leq 1)$. As shown in the next section, this allows to introduce Markov Process (MP) and Stochastic Differential Equation (SDE) theories that will provide a simple method for deriving the asymptotic results. Substituting (2) into (1), the $E_{\mathrm{s}} / N_{\mathrm{t}}$ conditioned on a level of previous interference $\xi$ tends to the following limit when $K, N \rightarrow \infty, k / K \rightarrow t$ and $1 / K \rightarrow \mathrm{d} t$,

$$
\begin{aligned}
\Gamma(t \mid \xi) & \doteq \lim _{K \rightarrow \infty} \Gamma_{K}[k \mid \xi]=\frac{E_{s}(t)}{N_{0}+\xi(t)+\alpha \theta N_{\mathrm{rem}}(t)}(4) \\
N_{\mathrm{rem}}(t) & \doteq \lim _{K \rightarrow \infty} N_{\mathrm{rem}}[k]=\alpha \theta \int_{t}^{1} E_{s}(u) \mathrm{d} u
\end{aligned}
$$

with $E_{s}(t)$ the continuous arbitrary energy profile, $\xi(t)$ the continuous state modeling the residual random interference power $N_{\text {prv }}(t) \doteq \lim _{K \rightarrow \infty} N_{\text {prv }}[k]$, and $\alpha=K / N$ denoting the load factor. We can also define the asymptotic error probability $p_{1}(t \mid \xi)$ and the asymptotic p.d.f. of $\xi(t)$ as follows,

$$
\begin{aligned}
p_{1}(t \mid \xi) & \doteq \lim _{K \rightarrow \infty} p_{1}[k \mid \xi]=\operatorname{PER}[\Gamma(t \mid \xi)] \\
\mathrm{f}_{\xi}(\xi ; t) & \doteq \lim _{(K, \Delta \xi) \rightarrow(\infty, 0)} \frac{\operatorname{Pr}\left[\xi \leq N_{\text {prv }}[k]<\xi+\Delta \xi\right]}{\Delta \xi}
\end{aligned}
$$

As the error probability $p_{1}(t \mid \xi)$ depends on the previous residual interference, the PER at stage $t$ can be obtained as,

$$
\pi_{1}(t) \doteq \mathbb{E}_{\xi}\left[p_{1}(t \mid \xi)\right]=\int_{0}^{\infty} p_{1}(t \mid \xi) \cdot \mathrm{f}_{\xi}(\xi ; t) \mathrm{d} \xi
$$

and the SIC average packet error rate as,

$$
\overline{\operatorname{PER}}(\alpha)=\int_{0}^{1} \pi_{1}(u) \mathrm{d} u
$$

where evaluation of $\overline{P E R}(\alpha)$, which depends on the load factor $\alpha$ by way of $p_{1}(t \mid \xi)$, needs the calculation of the p.d.f. $\mathrm{f}_{\xi}(\xi ; t)$ derived in Appendix I. 


\section{Evaluation of The Asymptotic PER}

Appendix I shows that the p.d.f. $\mathrm{f}_{\xi}(\xi ; t)$ obeys the FokkerPlanck Differential Equation (FPDE) in (20). The theory of Stochastic Differential Equations (SDE) [6] states that, using the Ito integral, a Stochastic Dynamical System (SDS) may be defined as in (10), which evolves in $t$ with state $\xi(t)$ distributed according to the p.d.f. $\mathrm{f}_{\xi}(\xi ; t)$ solving the FPDE (and with initial conditions of the SDS matching boundary constraints of the FPDE). This SDS, with input white Gaussian noise (WGN) $\eta(t)$ of two-sided power spectral density $S_{\eta \eta}(f)=1$ and output $\xi(t)$, becomes,

$$
\mathrm{d} \xi(t) / \mathrm{d} t=a(\xi(t), t)+\sigma(\xi(t), t) \cdot \eta(t)
$$

with nonlinearities $a(\xi, t)=\bar{E}(\xi, t)$ and $\sigma(\xi, t)=\Sigma^{1 / 2}(\xi, t)$ given in (21). In the limit, when $K, N \rightarrow \infty$, the diffusion term $\sigma(\xi(t), t)$ in (21) vanishes, and $\xi(t)$ fulfils the SDE,

$$
\mathrm{d} \xi(t) / \mathrm{d} t=\alpha \theta E_{s}(t)\left(\epsilon+(1-\epsilon) p_{1}(t \mid \xi(t))\right)
$$

which becomes deterministic provided that $\xi(0)=0$ holds true. In consequence, (11) becomes an Ordinary Differential Equation (ODE) with initial condition $\xi(0)=0$. If this ODE is solved for $\xi(t)=\check{\xi}(t)$, we have from (8) that $\pi_{1}(t)=\mathbb{E}_{\xi(t)}\left[p_{1}(t \mid \xi(t))\right]=p_{1}(t \mid \check{\xi}(t))=\operatorname{PER}[\Gamma(t \mid \check{\xi}(t))]$ and the average packet error rate over all SIC stages becomes,

$$
\overline{\operatorname{PER}}(\alpha)=\int_{0}^{1} \operatorname{PER}\left[\frac{E_{s}(t)}{N_{0}+\check{\xi}(t)+\alpha \theta \int_{t}^{1} E_{s}(u) \mathrm{d} u}\right] \mathrm{d} t
$$

for a DS-CDMA system with an asymptotically large number of users, load $\alpha=K / N$ kept constant and an arbitrary symbol energy profile $E_{s}(t)$ (regardless of whether users are ranked according to power or not). We remark, that the ODE for $\check{\xi}(t)$ cannot be solved explicitly and Euler's or higher order numerical methods must be used instead.

\section{Evaluation Algorithm for Finite $K$}

For finite $K, N_{\text {prv }}[k]$ no longer evolves deterministically and we must work with its discrete probability distribution $p_{\xi}[\xi ; k]=\sum_{i=1}^{n_{c}[k]} p_{i}[k] \delta\left(\xi-\xi_{i}[k]\right)$, with $n_{c}[k]=$ $2^{k-1}$ the total number of possible interference values at stage $k,\left\{\xi_{i}[k]\right\}_{1<i<n_{c}[k]}$ the set of interference values and $\left\{p_{i}[k]\right\}_{1 \leq i \leq n_{c}[k]}$ the associated probabilities. To compute the packet error probability, each interference value $\xi_{i}[k]$ has an associated packet error probability $Q_{i}[k]=p_{1}\left[k \mid \xi_{i}[k]\right]$ given by (3), with the same probability $p_{i}[k]$. Thus, the error probability at stage $k$ is $\pi_{1}[k]=\sum_{i=1}^{n_{c}[k]} p_{i}[k] Q_{i}[k]$. As $n_{c}[k]$ grows exponentially in $k$, the exact average PER: $\overline{P E R}=$ $\frac{1}{K} \sum_{k=1}^{K} \pi_{1}[k]$, would require intensive computations. Hence, we develop a particle-based algorithm [7] for approximating $\pi_{1}[k]$ with a fixed number $C$ of centroids/particles.

Let us define the $C$-particle set $\left\{\left(\widehat{p}_{i}[k], \widehat{Q}_{i}[k]\right), 1 \leq i \leq C\right\}$ with centroid $\widehat{Q}_{i}[k]=p_{1}\left[k \mid \widehat{\xi}_{i}[k]\right]$ associated with an equivalent interference $\widehat{\xi}_{i}[k]$, which is not necessarily in the set of possible values of $N_{\text {prv }}[k]$ determined by (2) and where equiprobable particles with $\widehat{p}_{i}[k]=1 / C$ are considered. The estimated $\pi_{1}[k]$ is $\widehat{\pi}_{1}[k]=\sum_{i=1}^{C} \widehat{p}_{i}[k] \widehat{Q}_{i}[k]$, where $\widehat{Q}_{i}[k]$ must be computed recursively from $\widehat{Q}_{i}[k-1]$. This is done by operating on the associated interference $\widehat{\xi}_{i}[k]$ as follows: for each particle $i$ at stage $k$, a packet decoding error/success occurs with probability $\widehat{Q}_{i}[k]$ and $1-\widehat{Q}_{i}[k]$, respectively, and these events result in the following two new interference levels $\widehat{\xi}_{i, 1}[k+1], \widehat{\xi}_{i, 0}[k+1]$ and corresponding probabilities,

$$
\begin{aligned}
\widehat{\xi}_{i, 1}[k+1]= & \widehat{\xi}_{i}[k]+\theta E_{s}[k] / N \\
& \widehat{p}_{i, 1}[k+1]=\widehat{p}_{i}[k] \cdot \widehat{Q}_{i}[k] \\
\widehat{\xi}_{i, 0}[k+1]= & \widehat{\xi}_{i}[k]+\epsilon \cdot \theta E_{s}[k] / N \\
& \widehat{p}_{i, 0}[k+1]=\widehat{p}_{i}[k] \cdot\left(1-\widehat{Q}_{i}[k]\right)
\end{aligned}
$$

producing a new set of $2 C$ non-equiprobable interference particles $\left\{\left(\widehat{p}_{i, \varepsilon}[k+1], \widehat{\xi}_{i, \varepsilon}[k+1]\right), 1 \leq i \leq C, \varepsilon=0,1\right\}$. The resulting set of equiprobable packet error rate particles $\left\{\left(\widehat{p}_{i}[k+1], \widehat{Q}_{i}[k+1]\right), 1 \leq i \leq C\right\}$ for the next iteration is computed as follows,

1) Sort the resulting set of $2 C$ non-equiprobable particles $\left\{\left(\widehat{p}_{i, \varepsilon}[k+1], \widehat{\xi}_{i, \varepsilon}[k+1]\right), 1 \leq i \leq C, \varepsilon=0,1\right\}$ in ascending order w.r.t. $\xi$, yielding the ordered set of interference particles $\left\{\left(\widehat{p}_{j}[k+1], \widehat{\xi}_{j}[k+1]\right), 1 \leq j \leq 2 C\right\}$ where $j$ stands for the index in the ordered case.

2) Compute the corresponding packet error probability particles $\left\{\left(\widehat{p}_{j}[k+1], \widehat{Q}_{j}[k+1]\right), 1 \leq j \leq 2 C\right\}$, with $\widehat{Q}_{j}[k+1]=p_{1}\left[k \mid \widehat{\xi}_{j}[k+1]\right]$.

3) From $\left\{\left(\widehat{p}_{j}[k+1], \widehat{Q}_{j}[k+1]\right), 1 \leq j \leq 2 C\right\}$, perform resampling [7] to obtain the set of equiprobable particles $\left\{\left(\widehat{p}_{i}[k+1], \widehat{Q}_{i}[k+1]\right), 1 \leq i \leq C\right\}$ with $\widehat{p}_{i}[k+1]=1 / C$. The procedure is initialized at $k=1$ with null equiprobable interference particles $\left\{\left(\widehat{p}_{i}[1]=1 / C, \widehat{\xi}_{i}[1]=0\right), 1 \leq i \leq C\right\}$ and estimation of $\overline{P E R}$ is obtained from $\frac{1}{K} \sum_{k=1}^{K} \widehat{\pi}_{1}[k]$ where $\widehat{\pi}_{1}[k]=\sum_{i=1}^{C} \widehat{p}_{i}[k] \widehat{Q}_{i}[k]$. We stress that re-sampling the packet error probability particles $\left\{\left(\widehat{p}_{j}[k+1], \widehat{Q}_{j}[k+1]\right), 1 \leq\right.$ $j \leq 2 C\}$ rather than the associated set of residual interference particles $\left\{\left(\widehat{p}_{j}[k+1], \widehat{\xi}_{j}[k+1]\right), 1 \leq j \leq 2 C\right\}$ avoids numerical error propagation and is crucial to the accuracy of the method.

\section{Simulations And Conclusions}

To validate the asymptotic PER in (12), Figure 1 compares the empirical PER versus $\alpha\left(3 \cdot 10^{4}\right.$ Monte Carlo runs) among three spreading factors with the asymptotic PER in (12). Uniform sampling of the exponential profile $E_{s}(t) /\left.N_{0}\right|_{\mathrm{dB}}=$ $14-5 t$ and residual interference $\epsilon=0.1$ is set for all cases. The simulated FEC is the 3GPP Turbo Code with rate 1/3 and block length 256 QPSK symbols. As shown in Fig. 1, for increasing $K(K \geq 128)$, the empirical PER approaches the asymptotic solution, which is obtained solving the ODE in (11) using a standard numerical method.

Figure 2 analyzes the performance of the particle method in Section III $(C=32)$ for finite $K$, in comparison with a finite-user version of the SDS in (10) and with the empirical PER obtained with Monte Carlo. The SDS is computed using Euler's method for ODE's, setting the variance of the input Gaussian noise process $\eta[k]$ to $K$ : the discrete version for a single $\eta[k]$ realization becomes $\xi[k+1]=\xi[k]+$ $\left.\frac{1}{K}(a(\xi[k], k])+\sigma(\xi[k], k) \cdot \eta[k]\right)$, where the PER is averaged over $10^{3}$ realizations of $\eta[k]$. In Figure 2, the particle-based method closely fits the empirical PER, while the SDS-based 


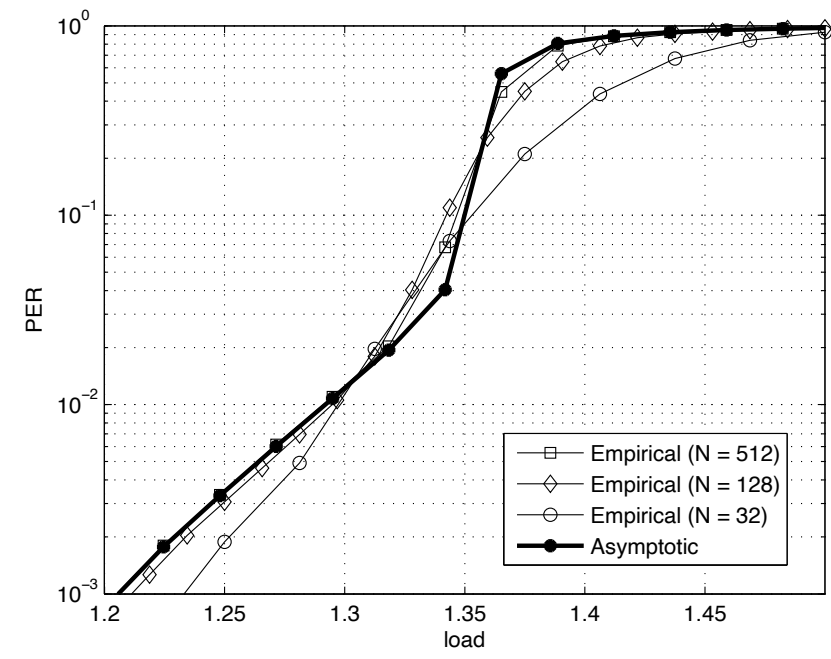

Fig. 1. Asymptotic and Empirical PER vs. load $\alpha=K / N$.

method, extrapolated to finite $K$ with diffusion the expression within the limit in (21), is comparatively less precise at low spreading factors (although more accurate for lower $\epsilon$ 's). We conclude thus that the particle method in Sec. III is preferable and provides fast and precise approximations to the PER for finite $K$, avoiding computationally expensive Monte Carlo evaluations. Also, the asymptotic average PER of a many-user DS-CDMA SIC has been derived under the long code model for any energy profile $E_{\mathrm{S}}(t)$, allowing accurate performance evaluation even in scenarios subject to disparate user powers and operating with/without power control strategies. Recent adaptive DS-CDMA IC schemes addressing error propagation may be found in [8].

\section{APPENDIX I. EVALUATION OF $\mathrm{f}_{\xi}(\xi ; t)$}

For finite $K$ and from (2), the transition of $N_{\text {prv }}$ from stage $k$ to $k+1$ follows $N_{\text {prv }}[k+1]=N_{\text {prv }}[k]+(\theta / N) \epsilon[k] E_{s}[k]$, with $\epsilon[k] \in\{\epsilon, 1\}$ the random variable related with the succesful/unsuccesful decoding at the $k$-th stage. Then, the Chapman-Kolmogorov equation [6] from $t$ to $t+\mathrm{d} t$, with $1 / K \rightarrow \mathrm{d} t$ and $\theta E_{s}[k] / N \rightarrow \mathrm{d} \xi$, is expressed using the transition probabilities $p_{1}(t \mid \xi)$ and $1-p_{1}(t \mid \xi)$ in (3) as,

$$
\begin{gathered}
\quad \mathrm{f}_{\xi}(\xi ; t+\mathrm{d} t)=p_{1}(t \mid \xi-\mathrm{d} \xi) \cdot \mathrm{f}_{\xi}(\xi-\mathrm{d} \xi ; t)+ \\
+\quad\left(1-p_{1}(t \mid \xi-\epsilon \cdot \mathrm{d} \xi)\right) \cdot \mathrm{f}_{\xi}(\xi-\epsilon \cdot \mathrm{d} \xi ; t)
\end{gathered}
$$

where $\mathrm{d} \xi=\alpha \theta E_{s}(t) \mathrm{d} t$ is the differential interference power increment associated with an error event at $t$. Setting $p_{1} \doteq$ $p_{1}(t \mid \xi)$ and $\mathrm{f}_{\xi} \doteq \mathrm{f}_{\xi}(\xi ; t)$, and defining either $\beta=1$ or $\beta=\epsilon$, we apply a 2 nd-order Taylor expansion in $\xi$ to (17), where $p_{1}(t \mid \xi-\beta \mathrm{d} \xi) \cdot \mathrm{f}_{\xi}(\xi-\beta \mathrm{d} \xi ; t)$ is approximated by $p_{1} \mathrm{f}_{\xi}+\partial_{\xi}\left[p_{1} \mathrm{f}_{\xi}\right](-\beta \mathrm{d} \xi)+\frac{1}{2} \partial_{\xi}^{2}\left[p_{1} \mathrm{f}_{\xi}\right](\beta \mathrm{d} \xi)^{2}$, with $\partial_{\xi}[\cdot]$ the partial derivative in $\xi$. Then, setting $\mathrm{f}_{\xi}^{+} \doteq \mathrm{f}_{\xi}(\xi ; t+\mathrm{d} t)$, we get,

$$
\begin{aligned}
& \mathrm{f}_{\xi}^{+} \simeq p_{1} \mathrm{f}_{\xi}+\left(1-p_{1}\right) \mathrm{f}_{\xi}+\partial_{\xi}\left[\mathrm{f}_{\xi}\right](-\epsilon \mathrm{d} \xi)+\frac{1}{2} \partial_{\xi}^{2}\left[\mathrm{f}_{\xi}\right](\epsilon \mathrm{d} \xi)^{2}+ \\
& +\partial_{\xi}\left[p_{1} \mathrm{f}_{\xi}\right] \cdot(-1+\epsilon) \mathrm{d} \xi+\frac{1}{2} \partial_{\xi}^{2}\left[p_{1} \mathrm{f}_{\xi}\right]\left(1-\epsilon^{2}\right)(\mathrm{d} \xi)^{2}
\end{aligned}
$$

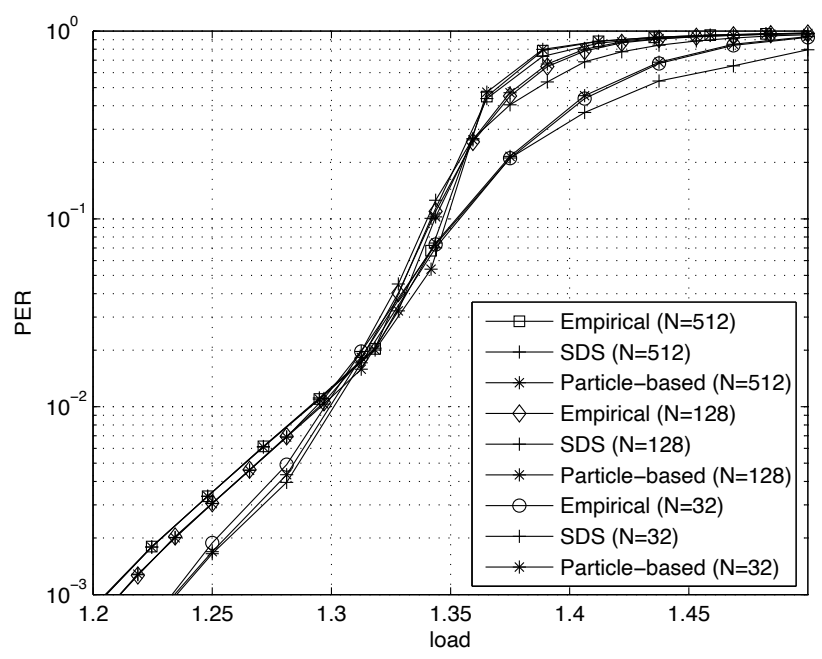

Fig. 2. Empirical PER vs. load $\alpha=K / N$ compared with the SDSand particle-based approximations.

As $\mathrm{d} \xi=\alpha \theta E_{s}(t) \mathrm{d} t$ is independent of $\xi$, we group $\partial_{\xi}^{i}[\cdot]$ terms and, setting $\Delta \mathrm{f}_{\xi} \doteq \mathrm{f}_{\xi}^{+}-\mathrm{f}_{\xi}=\mathrm{f}_{\xi}(\xi ; t+\mathrm{d} t)-\mathrm{f}_{\xi}(\xi ; t)$, we get,

$$
\begin{aligned}
\Delta \mathrm{f}_{\xi} & \simeq-\partial_{\xi}\left[\left(\left(\epsilon+(1-\epsilon) p_{1}\right) \alpha \theta E_{s}(t) \cdot \mathrm{f}_{\xi}\right] \mathrm{d} t+\right. \\
& +\frac{1}{2} \partial_{\xi}^{2}\left[\left(\left(\epsilon^{2}+\left(1-\epsilon^{2}\right) p_{1}\right) \alpha^{2} \theta^{2} E_{s}^{2}(t) \cdot \mathrm{f}_{\xi}\right](\mathrm{d} t)^{2}\right.
\end{aligned}
$$

Dividing now (19) by $\mathrm{d} t$, and using $\alpha=K / N$ and $\mathrm{d} t=1 / K$ within $\partial_{\xi}^{2}[\cdot]$, we get for $K, N \rightarrow \infty$ a Fokker-Planck (FP) Partial Differential Equation (PDE) [6],

$$
\partial_{t}\left[\mathrm{f}_{\xi}\right]=-\partial_{\xi}\left[\bar{E} \cdot \mathrm{f}_{\xi}\right]+\frac{1}{2} \partial_{\xi}^{2}\left[\Sigma \cdot \mathrm{f}_{\xi}\right]
$$

with drift $\bar{E}$ and diffusion $\Sigma$ given by,

$$
\begin{aligned}
& \bar{E}=\bar{E}(\xi, t)=\alpha \theta E_{s}(t) \cdot\left(\epsilon+(1-\epsilon) p_{1}(t \mid \xi)\right) \\
& \Sigma=\Sigma(\xi, t)=\lim _{N \rightarrow \infty}\left[\alpha \frac{\theta^{2} E_{s}^{2}(t)}{N} \cdot\left(\epsilon^{2}+\left(1-\epsilon^{2}\right) p_{1}(t \mid \xi)\right)\right]
\end{aligned}
$$

\section{REFERENCES}

[1] S. Verdu, S. Shamai, "Spectral Efficiency of CDMA with Random Spreading," IEEE Trans. on Information Theory, vol. 45, no. 2, Mar. 1999.

[2] D.N.C. Tse, S.V. Hanly, "Linear Multiuser Receivers: Effective Interference, Effective Bandwidth and User Capacity," IEEE Trans. on Information Theory, vol. 45, no. 2, Mar. 1999.

[3] D. Djonin, V. K. Bhargava, "Asymptotic Analysis of the Conventional Decision Feedback Receiver in Fading Channels," IEEE Trans. on Wireless Commun., Sep. 2003.

[4] A. Agrawal, J.G. Andrews, J.M. Cioffi, T.H. Meng, "Iterative power control for imperfect successive interference cancellation," IEEE Trans. on Wireless Comm., vol.4, no.3, May 2005.

[5] R.M. Buehrer, "Equal BER performance in linear successive interference cancellation for CDMA systems," IEEE Trans. on Commun., vol.49, no.7, Jul. 2001.

[6] H. Risken, The Fokker-Planck Equation: Methods of Solutions and Applications (2nd edition), Springer, 1989.

[7] P.M. Djuric, J.H. Kotecha et al., 'Particle filtering," IEEE Signal Processing Magazine, vol.20, no.5, Sep. 2003.

[8] R.C. de Lamare, R. Sampaio-Neto, "Minimum Mean-Squared Error Iterative Successive Parallel Arbitrated Decision Feedback Detectors for DS-CDMA Systems", IEEE Trans. on Communications, vol.56, no.5, May 2008. 